\title{
Influence of Hormone Supplementation Therapy on the InCidence of Denture Stomatitis and on Chemiluminescent ACtivity of Polymorphonuclear Granulocytes in Blood of Menopausal-Aged WOMEN
}

\author{
M. Golecka-Bakowska, E. Mierzwinska-Nastalska, M. Bychawska \\ Department of Prosthodontics, Warsaw Medical University, Warsaw, Poland; Laboratory Diagnostics Unit, National Institute of Tuberculosis \\ and Lung Diseases, Warsaw, Poland
}

\begin{abstract}
Background: Menopause is a health and social problem that affects a large number of women. Inadequate quantity of steroid hormones also impacts quality of the mucous membrane of the oral cavity. During menopausal age, many women wear removable prosthetic restorations in order to replace missing teeth. Such restorations may facilitate the development of inflammations in the surface of the oral cavity, referred to as denture stomatitis.

Objective: The aim of the study was to evaluate the influence of hormone supplementation therapy on the incidence of Candida-associated denture stomatitis and on the metabolic activity of polymorphonuclear granulocytes in peripheral blood of female patients.

Material and methods: The study was conducted on a group of women in menopausal age, users of hormone replacement therapy and of removable prosthetic restorations. Female patients were subjected to a clinical study that included interviews and physical examinations. Laboratory microbiological examinations were completed on the basis of direct swabs collected from the mucous membrane of the oral cavity and from the surface of dentures. Metabolic activity of polymorphonuclear granulocytes in peripheral blood of female patients was evaluated by means of a chemiluminescence test.

Results: Candida-associated denture stomatitis observed was characterized by a strong growth of fungi and a lower chemiluminescent activity of neutrophils in blood of female patients undergoing hormone supplementation therapy.

Conclusions: Patients using hormone supplementation therapy and removable prosthetic restorations constitute a high-risk group for Candida infections and inflammations of the mucous membrane of the oral cavity; thus they should remain under constant dental control.
\end{abstract}

Key words: denture stomatitis, menopausal women, hormonal replacement therapy

\section{INTRODUCTION}

The menopause is a natural, genetically-programmed physiological process that takes place when ovaries re- tire, leading to a shortage of estrogens. That, in turn, becomes the cause of many resulting symptoms that destabilize the psychological and physical condition of a woman and result in a series of health issues: vascular and motor problems, disorders of the urinary and sexual systems, nutrition dysfunctions of skin and connective tissue, osteoporosis, as well as heart and vascular diseases $[1,2]$. Shortage of steroid hormones also influences the condition of the mucous membrane in the oral cavity. Inflammations cause irritations of the mucous membrane, the sensation of dryness and burning, taste disorders and proneness to infections. Application of hormone supplementation therapy, in the form of estrogens or joint estrogenprogesterone therapy, limits the disadvantageous symptoms of the menopause [3].

Receptors of sex hormones are located in many tissues, including cells involved in immunological reactions. Estrogens applied as part of hormone supplementation therapy act as negative regulators of lymphopoiesis that limit the production of precursors of B-lymphocytes, stimulated by mitogens. A similar condition can be observed during pregnancy [4]. Kamada et al [5] have proven that hormone supplementation therapy causes increased concentration of M-CSF in blood with no influence on the level of TGF- $\beta$, TNF$\alpha$, GM-CSF, and IL-18. Estrogen receptors have been recognized also on the surface of neutrophils that produce nitric oxide. It has been observed that following a therapy with $17-\beta$-estradiol an increase in nitric oxide production by neutrophils takes place. Such a situation may facilitate inflammatory processes in that these cells actively participate $[6,7]$.

The term denture stomatitis describes inflammatory changes of the mucous membrane of the oral cavity, caused by wearing prosthetic restorations, especially removable ones made of an acrylic material [8, 9]. Available publications and our own research indicate that denture stomatitis can be observed in 36 to $67 \%$ of cases, often associated with a fungal infection. In most cases, the isolated fungi represent the Candida species $[8,10,11]$. The resulting ailments may cause the sensations of burning and dryness, as well as pain and taste disorders.

Development of denture stomatitis may be promoted by general and local factors. According to the 
literature, the most important local factors are: traumatic results of using dentures, an infection with yeast-like fungi, and the presence of denture plaque $[8$, 12]. General ailments that hinder immunity of the mucous membrane to traumas and promote the development of yeast-like fungi include: hormonal disorders (diabetes, menopause), cases of cancer, vitamin deficiency, and nourishment deficiencies [13-15]. It is believed that denture somatitis results from a combined influence of many factors $[11,14,15]$.

The aim of the study was to evaluate the influence of hormone supplementation therapy on the incidence of denture stomatitis and to evaluate the chemiluminescent activity of polymorphonuclear granulocytes in peripheral blood of female patients who undergo hormone supplementation therapy and use removable prosthetic restorations.

\section{Material And Methods}

The study was performed in accordance with the Declaration of Helsinki for Human Research and study protocol was approved by the Ethics Committee of Warsaw Medical University in Warsaw, Poland.

The study was conducted on a group of 130 women in menopausal age, users of removable prosthetic restorations. The group was composed of $65 \mathrm{fe}-$ male patients aged 44 to 59 (mean age: 51.9 years), wearers of removable prosthetic restorations who had been undergoing hormone supplementation therapy for at least 1 year (administered orally or through the skin). Members of the study group did not take other medications and remained in good health, with no overt causes of hindered immunity. The control group included 65 female patients, aged 47 to 57 (mean: 54 years). These women were generally healthy, wore prosthetic restorations and did not undergo hormone supplementation therapy.

Clinical study included interviews and physical examinations of the mucous membrane in the oral cavity, as well as the evaluation of dentures. Subjective syndromes concerning the oral cavity were registered (pain, dryness, burning of the mucous membrane and taste disorders). Inflammation of the mucous membrane of the prosthetic base was evaluated according to Newton's classification [9]: from a limited local inflammation to general inflammation of the whole mucous membrane area that is covered by a denture.

Mycological examination was executed on the basis of direct swabs from the mucous membrane of the oral cavity. Swabs were collected with the help of a sterile cotton ball and sent to a laboratory for preparation of a direct smear and for inoculation on a Sabouraud agar. Positive result of the serum filamentation test identified the species Candida albicans. Biochemical qualities of particular strains were checked by means of the API Candida test (BioMerieux, France). In the latter part of the study, mycological study results were analyzed in a qualitative and quantitative manner.

In order to rate polymorphonuclear granulocytes in peripheral blood of menopausal-aged women, venous blood was collected from 31 patients in the study group and 31 in the control group. Next, neutrophils were analyzed and the chemiluminescence test was executed on the collected samples. The incidence of polymorphonuclear granulocytes was checked by conducting the May-Grunwald-Giemsa staining procedure. Chemiluminescent activity (CL) of polymorphonuclear granulocytes was assessed by means of the modified Easmon and Cole method in venous blood suspension that was merged with PBS solution, with the addition of glucose and bovine albumin. To strengthen the chemiluminescence effect, cellular suspension was merged with luminol solution (Sigma, St. Louis, MO). Next, resting chemiluminescence was measured in the $3 \mathrm{H}$ channel for a couple of minutes. Once resting chemiluminescence was established, FMLP solution was added to blood suspension (Sigma, St. Louis, MO) in PBS and CL of polymorphonuclear granulocytes was gauged. Chemiluminescence measurements were displayed in the CPM form (light impulses per minute). Final results were presented as the maximum value of the chemiluminescence measurement $\left(\mathrm{CL}_{\max }\right)$ divided by the standard value of 1000 polymorphonuclear granulocytes.

Results were processed with the help of statistical methods in order to facilitate conclusions regarding qualitative and quantitative relations between particular parameters. Statistical analysis included mean values (for quantitative qualities), standard deviation, standard error, and the percentage ratio. Chemiluminescent activity was presented as the mean activity value $-\mathrm{CL}_{\max }$.

\section{RESULTS}

As a result of physical examinations conducted on hormone supplementation therapy patients, in 29.2\% of cases, subjective symptoms in the form of dryness (Chi-square $=4.5 ; \mathrm{P}<0.05)$ and burning $(24.6 \%)$ in the oral cavity (Chi-square $=8.5 ; \mathrm{P}<0.05$ ) were observed. Patients who did not receive hormone supplementation therapy often complained about pain $(23 \%)$ (Chisquare $=2.1 ; \mathrm{P}>0.05)$ and taste disorders $(13.9 \%)$ within the oral cavity (Chi-square $=3.1 ; \mathrm{P}>0.05$ ), but these differences were not statistically significant (Table 1). During clinical evaluation, condition of the mucous membrane on the prosthetic base was checked according to the modified Newton's classification. Inflammation was more often recognized in the group of hormone supplementation therapy users (Chisquare $=17.7 ; \mathrm{P}<0.05)$.

When results of the mycological examination were analyzed, a significantly larger mean number of fungi colonies was observed in case of Candida albicans

Table 1. Subjective symptoms in the oral cavity (\%).

\begin{tabular}{c|cccc}
\hline & \multicolumn{4}{|c}{ Subjective symptoms } \\
\cline { 2 - 5 } & Pain & Dryness & Burning & Taste disorders \\
\hline $\begin{array}{c}\text { Study group } \\
(\mathrm{n}=65)\end{array}$ & 10.8 & 29.2 & 24.6 & 6.2 \\
\hline $\begin{array}{c}\text { Control group } \\
(\mathrm{n}=65)\end{array}$ & 23 & 13.8 & 6.2 & 13.9 \\
\hline
\end{tabular}


Table 2. Results of the mycological examination based on swabs collected from mucous membrane surface of the oral cavity.

\begin{tabular}{|c|c|c|c|c|c|c|c|c|}
\hline & \multicolumn{2}{|c|}{ Candida albicans } & \multicolumn{2}{|c|}{ Candida glabrata } & \multicolumn{2}{|c|}{ Candida krusei } & \multicolumn{2}{|c|}{ Candida arapsilosis } \\
\hline & $\begin{array}{l}\text { Fungal } \\
\text { presence }\end{array}$ & $\begin{array}{l}\text { Fungal } \\
\text { colonies }\end{array}$ & $\begin{array}{c}\text { Fungal } \\
\text { presence }\end{array}$ & $\begin{array}{l}\text { Fungal } \\
\text { colonies }\end{array}$ & $\begin{array}{c}\text { Fungal } \\
\text { presence }\end{array}$ & $\begin{array}{l}\text { Fungal } \\
\text { colonies }\end{array}$ & $\begin{array}{c}\text { Fungal } \\
\text { presence }\end{array}$ & $\begin{array}{l}\text { Fungal } \\
\text { colonies }\end{array}$ \\
\hline $\begin{array}{l}\text { Study group } \\
\quad(\mathrm{n}=65)\end{array}$ & $61.5 \%$ & $10.6 \pm 2.7$ & $16.9 \%$ & $1.1 \pm 0.4$ & $3.1 \%$ & $0.06 \pm 0.04$ & $4.6 \%$ & $0.06 \pm 0.03$ \\
\hline $\begin{array}{l}\text { Control group } \\
\qquad(\mathrm{n}=65)\end{array}$ & $50.8 \%$ & $2.5 \pm 0.6$ & $18.5 \%$ & $0.2 \pm 0.0$ & $6.2 \%$ & $0.22 \pm 0.11$ & $3.1 \%$ & $0.50 \pm 0.10$ \\
\hline
\end{tabular}

Fungal colonies are reported as means \pm SD.

Table 3. Level of chemiluminescence activity (CL) of polymorphonuclear granulocytes.

\begin{tabular}{c|c|c|c}
\hline & $\mathrm{CL}_{\max }(\mathrm{CPM})$ & White blood cells & Neutrophils (\%) \\
\hline Study group & $26487.5 \pm 2991.8$ & $6.2 \times 10^{9} \pm 0.12 \times 10^{9}$ & $60.2 \pm 0.6$ \\
Control group & $33314.5 \pm 3683.7$ & $6.2 \times 10^{9} \pm 0.15 \times 10^{9}$ & $54.6 \pm 0.7$ \\
\hline
\end{tabular}

Values are means $\pm \mathrm{SE}$.

$(t$-test $=2.8 ; \mathrm{P}<0.05)$ and Candida glabrata $(\mathrm{t}$-test $\mathrm{t}=$ $2.1 ; \mathrm{P}<0.05)$ in comparison with the control group (Table 2). This examination was based on swabs collected from the mucous membrane surface of the oral cavity.

In the group of women using hormone supplementation therapy, lower CL of blood polymorphonuclear granulocytes in comparison with the control group was observed. The mean value of $\mathrm{CL}_{\max }$ of polymorphonuclear granulocytes in the study group was 26487.5 \pm 2991.8 (SE). In the control group, not subjected to hormone supplementation, the mean $\mathrm{CL}_{\max }$ was $33314.5 \pm 3683.7$ (Table 3). When marking the chemiluminescent activity of blood polymorphonuclear granulocytes, their number in the study group remained between 54.4 and $63.7 \%$ of blood corpuscles, while in the control group it remained between 49.5 and $64.1 \%$ of blood corpuscles (Table 3 ).

Comparing the results for particular female patients directly, a relation was noted between the increase in intensity of fungi colony growth indicated during the mycological examination and the CL activity level of blood granulocytes in laboratory examination.

\section{Discussion}

Clinical observations suggest that many women in menopausal age use prosthetic restorations. Lower steroid hormone concentration influences the condition of the mucous membrane of the oral cavity, often leading to irritations of the mucous membrane, the sensation of dryness, burning, taste disorders and proneness to infections [2]. Results of research completed in preparation of this paper suggest that female patients who did not undergo hormone supplementation therapy often complained about pains and taste disorders, while women subjected to hormone supplementation therapy more often reported subjective ailments such as dryness and burning, which could be at- tributed to a more intensive growth of yeast-like fungi within the oral cavities of these patients.

The presence of dentures in the oral cavity, especially when the denture base is supported on a vast surface, creates unique non-physiological conditions. Self-cleaning by saliva is hindered by the denture base, while increased temperature and humidity facilitate bacteria and fungi growth $[8,12]$. The danger of a fungal infection depends on risk factors observed in a given patient. Apart from local factors in the oral cavity related to the usage of dentures, condition of the host's immunological system plays a significant role. In mycological studies for this paper in a group of patients undergoing hormone supplementation therapy, a significantly higher concentration of fungi growth was identified. Metabolic products excreted by the intensively growing fungal cells and elements of fungal cellular walls effecting the environment $[8,16]$ could intensify the manifestation of clinical symptoms in the form of the inflammation of the mucous membrane of the oral cavity, as well as subjective syndromes burning, and dryness - in the group of patients undergoing hormone supplementation therapy.

Fungi growth and infection development begins with selective adhesion to the mucous membrane of the host. Among all fungi of the Candida type, the greatest degree of adhesion to epithelium cells is displayed by Candida albicans species. Mannoproteins, chitin, proteins and lipids that enter into reactions with receptors in the mucous membrane act as adhesins [17]. A major role in anti-fungal defense is played by the reticuloendothelial system.

Hindrance of the oxygen-based mechanism of bacteria elimination by polymorphonuclear granulocytes can be observed during granulomatous disease, as well as primary and secondary granulocytopenia [18]. Such patients display a greater proneness to fungal infections [17]. Pietruski et al [19] have concluded that in patients with diagnosed denture stomatitis with 
fungal infection polymorphonuclear granulocytes in blood show limited reticuloendothelial activity. Research for this paper concerning the evaluation of the activity of reticuloendothelial cells in eliminating bacteria and fungi, with the application of a chemoluminescence test, indicated a lower metabolic activity of polymorphonuclear granulocytes in blood of patients undergoing hormone supplementation therapy. As studies by other authors show, hormone supplementation therapy may lower the production of highly-reactive oxygen derivatives by peripheral blood neutrophils, mainly through a direct operational mechanism of estrogens applied in the therapy. Neutrophils feature estrogen receptors on their surface. By interacting with these receptors, estrogens trigger a biological effect by influencing the expression of myeloperoxidase and nitrous oxide. Pertynska et al [7] have shown that, when an estradiol-progesterone substance is applied, hormone supplementation therapy modifies the production of reactive oxygen forms by peripheral blood neutrophils. These authors have argued that the substance used limits the production of free radicals, in both in vitro and in vivo conditions. Bekesi et al [20] believe that hormone therapy reverses the decline in myeloperoxidase activity of polymorphonuclear granulocytes. This decline takes place after the menopause, in comparison with myeloperoxidase activity in female organisms in the reproductive period.

Analysis of the present results revealed a higher growth of fungi disclosed by the mycological examination in the group of patients subjected to hormone supplementation therapy. It is possible that the increased growth of fungi flora in these patients may remain in connection with the limited chemiluminescent activity of polymorphonuclear granulocytes in blood, after the application of hormone therapy.

\section{CONCLUSIONS}

1. Patients using hormone supplementation therapy and removable prosthetic restorations constitute a high-risk group in terms of fungal infections and inflammations of the mucous membrane of the oral cavity, thus they must remain under constant dental control in order to avoid the appearance of denture stomatitis with fungal infections.

2. Chemiluminescent activity of polymorphonuclear granulocytes in peripheral blood of patients subjected to hormone supplementation therapy is lower in comparison with the control group. This suggests a potential influence of hormone supplementation therapy of the metabolic activity of granulocytes. That, in turn, may hinder anti-fungal defenses and facilitate fungi growth.

Conflicts of interest: No conflicts of interests were declared by the authors in relation to this article.

\section{REFERENCES}

1. Baron J. Overview of Gynecological and Obstetric Endocrinology. Warsaw, PZWL, 1996 (Textbook in Polish)

2. Skalba P. Hormonal Replacement Therapy. Warsaw, PZWL, 2002 (Textbook in Polish).
3. Friedlander AH. The physiology, medical management and oral implications of menopause. J Am Dent Assoc 2002; 133: 73-81.

4. Stites DP, Siiteri PK. Steroids as immunosuppressants in pregnancy. Immunol Rev 1983; 75: 117-38.

5. Kamada M, Irahara M, Maegawa M, Ohmoto Y, Takeji T, Yasui T, Aono T. Postmenopausal changes in serum cytokine levels and hormone replacement therapy. Am J Obstet Gynecol 2001; 184: 309-14.

6. Garcia-Duran M, de Frutos T, Diaz-Recasens J, GarciaGalvez G, Jimenez A, Monton M, Farre J, Sanchez de Miguel L, Gonzalez-Fernandez F, Arriero MD, Rico L, Garcia R, Casado S, Lopez-Farre A. Estrogen stimulates neuronal nitric oxide synthase protein expression in human neutrophils. Circ Res 1999; 85: 1020-6.

7. Pertynska MP, Tchorzewski H, Lewkowicz P, Pasnik J, Pertynski T. Evaluation of the hormonal replace therapy (HRT) effect on generation of reactive oxygen intermediates (ROI) by neutrophil in peripheral blood of menopausal women. Ginkol Pol 2000; 71: 55-62.

8. Spiechowicz E, Mierzwinska-Nastalska E. Fungi of the Oral Cavity. Warsaw, Med Tour Press Int, 1998 (Article in Polish).

9. Newton A. Denture sore mouth. Brit Dent J 1962; 112: 357-60.

10. Dorko E, Jenca A, Pilipcinec E, Danko J, Svicky E, Tkacikova L. Candida associated denture stomatitis. Folia Microbiol 2001; 46(5): 443-6.

11. Jacopino AM, Wathen WF. Oral candidal infection and denture stomatitis: A comprehensive review. JADA 1992; 123: 46-51.

12. Budtz-Jörgensen E, Mojon E, Banon-Clement J M, Baehni P. Oral candidosis in long-term hospital care: Comparison of edentulous and dentate subjects. Oral Dis 1996; 2: 285-90.

13. Budtz-Jörgensen E. Etiology, pathogenesis, therapy and prophylaxis of oral yeast infections. Acta Odontol Scand 1990; 48: 61-9.

14. Dorocka-Bobkowska B, Budtz-Jörgensen E, Wloch S. Non-insulin-dependent diabetes mellitus as a risk factor for denture stomatitis. J Oral Pathol Med 1996; 25; 411-5.

15. Samaranayake LP. Oral candidosis: an old disease in new guises. Dent Update 1990; 17: 36-8.

16. Dzierżanowska D. Fungi infections; the pathogenesis, clinic, and therapy. Terapia 1998; 2: 3-8 (Article in Polish).

17. Cohen MS, Isturits RE, Malech HL, Root RK, Wilfert $\mathrm{CM}$, Gutman L, Buckley RH. Fungal infection in chronic granulomatous disease. Am J Med 1981; 71: 59-66.

18. Robinson P, Walkefield D, Breit SN, Easter JF, Penny R. Chemiluminescent response of pathogenic organisms: normal human polymorphonuclear leukocytes. Infect Immun 1984; 43: 744-52.

19. Pietruski J, Jabłonska E, Stokowska W. Functional deficiency of peripheral blood neutrophils in persons with denture stomatopathy complicated with fungal infection. Czasop Stom 1997; 1: 10-15 (Article in Polish).

20. Bekesi G, Kakucs R, Varbiro S, Feher J, Pazmany T, Magyar Z, Sprintz D, Szekacs B. Induced myeloperoxidase activity and related superoxide inhibition during hormone replacement therapy. BJOG 2001; 108(5): 474-81.

Address for correspondence:

Magdalena Golecka-Bakowska

59, Nowogrodzka St.

02-006 Warsaw

Poland

Phone: +48 225021886

E-mail: goleckam@hotmail.com 